\title{
A Novel Architecture for 5G Ultra Dense Heterogeneous Cellular Network
}

\author{
Sabeen Tahir \\ Information Technology Department, Faculty of Computing and Information Technology \\ King Abdulaziz University \\ Jeddah 21589, Saudi Arabia
}

\begin{abstract}
The mounting use of wireless devices and wide range applications in an ultra-dense heterogeneous wireless network has directed to the challenging circumstances that could not be handled till 4G. In order to deal with the critical challenges, the fifth generation (5G) wireless network architecture requires an efficient well-organized wireless network. In this paper, a novel architecture for the 5G ultradense heterogeneous cellular network is proposed. In the proposed architecture two main aspects, massive MIMOOFDMA and IP-based vertical handover are considered. In order to have full network coverage, the whole macro area network is divided into the microcells and each microcell is further divided into the smaller cells. The heterogeneity of different types of base stations (macro area network base station, micro-cell base station, and small cell base station) provides efficient network coverage. By reducing the area of the cell, the frequency efficiency and network coverage are improved. All the base stations are equipped with the massive MIMO-OFDMA antennas and different radio access technologies so a single wireless device can switch from one radio access technology to the other. In order to prevent the link disconnection and save IP address, whenever wireless devices need to perform vertical handover first a new connection is established with new radio access technology. It is important to note the same IP address is used whereas the current connection is disconnected. By utilizing IP-based vertical handover, the new 5G wireless network can become the principal goal of service continuity and minimize the handover processing delay. The simulation results show the improvement in the network performance.
\end{abstract}

Keywords-Massive MIMO-OFDMA; 5 ; $; \quad$ IP-based interoperability; heterogeneous

\section{INTRODUCTION}

The inventive and efficient use of Information and Communication Technologies (ICT) is becoming more attractive and significant to advance the economy of the world. A wireless communication technology plays a role of the most critical element in the global ICT and it is a fast-growing trend in the world. The main requirements of the $5 \mathrm{G}$ system will have to include the simultaneous full-duplex transmission, use of massive multi-input - output (massive MIMO). In addition, millimeter wave communication, cloud-based radio access network and energy efficient technologies due to the energy starving multimedia applications [1]. Due to the promising trends of the frequency virtualization and energy efficient communication, the $5 \mathrm{G}$ network's structural design will be more complex than $4 \mathrm{G}$ network design [2, 3]. Finally, minimize delays in the network to improve the overall performance [4]. All the current mobile wireless networks are going to adapt the all-IP (Internet Protocol) principle, which means that all the data and signaling will be transmitted via IP (a unifying technology) on the internet layer [5, 6]. Recent years have seen incredible an enlargement in mobile data traffic and this trend is anticipated to continue in the future. To handle this enlargement, dense Transmission Points (TPs) e.g. Ultra-Dense Networks (UDNs) need to be deployed. The 5G cellular networks have to support the ubiquitous mobile broadband wireless access and advance the handling of extremely dense and heterogeneous networks. Fig. 1 shows an example of six typical UDN scenarios [7]. An extremely dense heterogeneous wireless network requires efficient resource management of applications and formation of efficient approach offering energy efficiency, scalability, and flexibility [8].

The upcoming $5 \mathrm{G}$ wireless networks will highly base to the highest degree of density and heterogeneity. Therefore, networks need to have an effective density and heterogeneity controlling techniques to handle the increasing amount of data traffic and wireless devices scattered in a skillful manner. The data traffic load of wireless network increases than the limit of future wireless networks. It is observed that an analogous connection exists between interference and scattered networks, where the whole throughput is not in line through the maximum scattered networks. Hence, the network scalability is a serious issue for the future $5 \mathrm{G}$ wireless network [9-11]. The energy efficiency can be monitored from the base stations and wireless mobile devices. From the base station perspective, it is worth controlling solutions that decrease energy consumption by connecting wireless devices into as few as the possible or frequent reconstruction of the power cycling method. Therefore, it requires an efficient technique that increases energy efficiency by getting an enhanced trade-off between the expected data traffic and existing energy. From the other perspective of wireless devices, the energy-efficient techniques maximize the energy efficiency. 

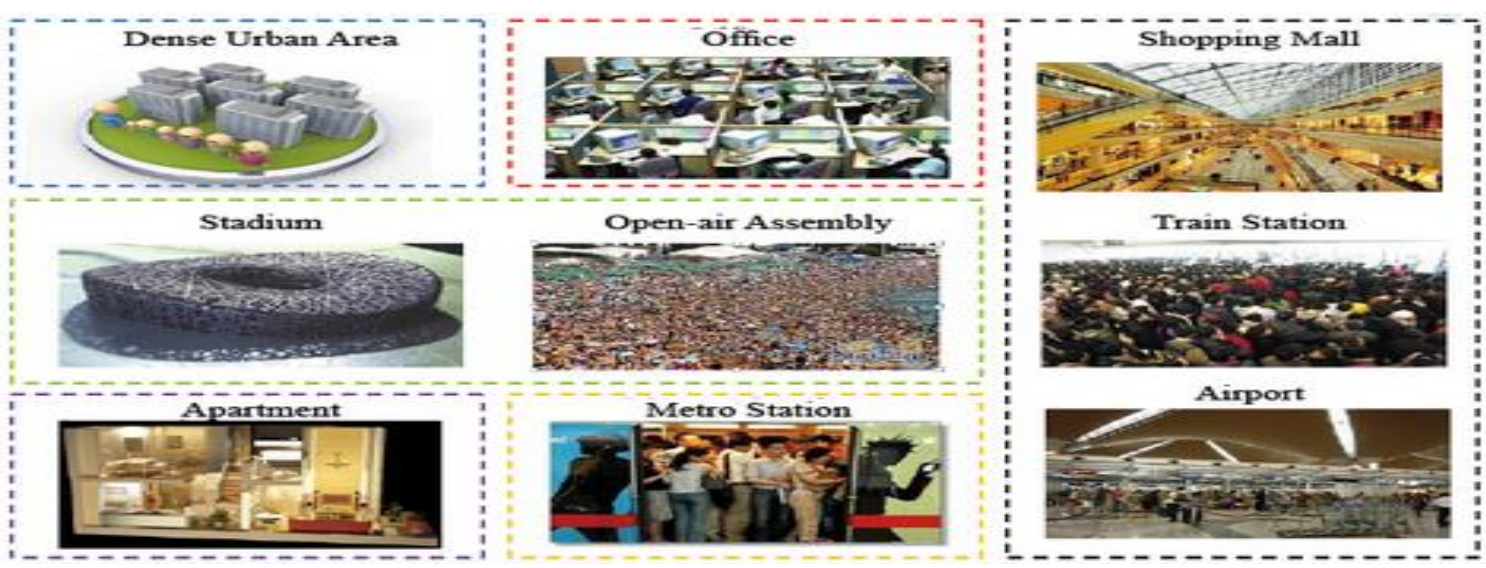

Fig. 1. Typical UDN Scenarios [7].

The future $5 \mathrm{G}$ extremely dense wireless network needs to improve the flexibility with an efficient control of energy consumption. Security is another perspective that conflicts with the scalability and energy efficiency. The wireless connections mostly interrupted due to unwanted interference or security attacks. These interruptions lead to some harmful effects with communication delay. It is an extremely challenging job to tackle scalability, flexibility, and energy competency at the same time as these requirements are in conflict with each other. The improvement in one can lead to the degradation of the other requirements. Suppose security is increased, but with the greater processing overhead, reduced scalability and energy efficiency. If the energy efficiency improves by switching off various network equipment, then the network scalability may be affected [12-14]. The proposed 5G network system will have the ability to control many base stations positioned dynamically in a heterogeneous manner. A macrocell overlaid with the microcells and small cells to carry the enhanced spectral efficiency and coverage within a network. The key assumptions of the proposed technique are 1) all the base stations are equipped with the massive MIMO-OFDMA antennas, 2) a user will have the ability to switch between different RATs without changing their IP address from a single wireless mobile device 3 ) the wireless devices can perform vertical handover efficiently without disconnecting their links.

This paper targets to develop a new operational design for the 5G network, Section 2 describes the related work and highlights the problems in current wireless techniques. In Section 3, the proposed $5 \mathrm{G}$ dense heterogeneous wireless network is explained. Simulation results are given in Section 4. Challenging in the $5 \mathrm{G}$ are discussed in Section 5. Finally, in Section 6 the future challenges and conclusions are drawn.

\section{RELATED WORK}

The use of extra frequency band will direct to an increasingly mobile frequency use in 5G. Advanced wireless techniques will facilitate drastically efficient frequency by using synchronized multipoint and small cell etc. For the sharing of frequency, a mobile architecture will allow the increase of multiplexing, the density of base stations and network heterogeneity. The previous generations 3G (UMTS and HSPA) and 4G (LTE and LTE-Advanced) focused on creating new radio transmission techniques. The $3 \mathrm{G}$ has the ability to handle complex data and support high data rates. The $4 \mathrm{G}$ network is expected to present comprehensive, secure internet access, gaming and streamed multimedia services. Now the research directs to the $5 \mathrm{G}$, in order to highlight the user-oriented challenges $5 \mathrm{G}$ network must focus on the network densification into an ultra-dense environment. It should enhance the features of mobile devices, including autointegration and self -management capabilities. Furthermore, high consistent connections put inflexible latency and reliability requirements on the structural design.

Multi-Input Multi-Output (MIMO) is a wireless radio antenna technique that utilizes multiple transmitters and receivers to broadcast more data at the same time. A base station of massive MIMO technique is equipped with a large number of antennas serving many wireless devices. It allows multiple antennas to transmit and receive multiple spatial streams and combine data streams receiving from different paths in order to increase the receiver signal capturing the power and capacity of a channel. MIMO played an imperative role in $3 \mathrm{G}$ and $4 \mathrm{G}$ wireless network $[15,16]$. Some other current techniques have designed the highly energy efficient massive MIMO [17]. The energy efficiency of a massive MIMO system depends on the circuit energy consumption. The best broadcast power increases with the number of antennas, so a broadcast power is a significant design parameter for high energy efficiency massive MIMO system [18]. Antennas are generally located on the surface of the carrier to get the wanted electromagnetic energy [19]. The MIMO technique has the ability to increase the throughput even under the conditions of signal fading, interference, and multipath [16, 20]. Millimeter wave communication is a potential 5G cellular network technique, which is expected to allow hundreds of $\mathrm{MHz}$ bandwidth [21]. The millimeter wave technology has some limitations such as not helpful for all kinds of wireless applications and millimeter wave technology is its limited range. According to the physics law "the shorter the wavelength, the shorter the communication range for a given power". By the side of logical power in many cases, the limitation contains the range less than 10 meters. The free space loss in $\mathrm{dB}$ can be calculated as below:

$L=92.4+90 \log (f)+20 \log (R)$ 
Where $R$ is considered as the Line-of-Sight (LoS) distance between transmitter and receiver antennas in kilometers, and $\mathrm{f}$ is the frequency in gigahertz. For instance, the loss at $10 \mathrm{~m}$ at $60 \mathrm{GHz}$ is:

$L=92.4+35.6-40=88 d B$

This loss can be overcome with good receiver intensity and high antenna's gain. The other limitation of the millimeter wave technique is atmospheres that can absorb it and limit its range. The signal attenuation is very high in the air due to the rain, fog, and humidity [22]. METIS has identified the 5G mobile communication scenarios. These situations reflect the predicted challenges like high-data-rate, accessibility, mobility, a huge amount of devices, low latency and consistency [23]. The objective of this project is only to combine the heterogeneous wireless technologies to optimize the range of radio devices. However, problems with this approach are, it does not provide the solution how to stable $5 \mathrm{G}$ requirements, which are contradicting each other. METIS's structural design mostly focused on hardware communication techniques. Although, the band utilization is mentioned it does not state anything regarding what type of technique will be in employment to reach an efficient and safe spectrum allocation [8].

A management architecture "Wireless Software-based architecture for Extremely Dense networks" (WiSEED) for the $5 \mathrm{G}$ system is proposed that handles three operational services routing, device mobility, and frequency usage. The aim of WiSEED is to handle the contradictory requirements such as scalability, flexibility, and energy efficiency in order to give a high quality and ubiquitous services for mobile broadband internet access in a dense environment. WiSEED focuses on the perspective of software networking, it occupies technologies and operational services based on the perception to support the envisage architecture. The recourse demand for all the advanced applications will be increased for the $5 \mathrm{G}$ network. The 5G network should have to advance the wireless transmissions and service rates like 4G. Therefore, 5G networks are supposed to permit transmissions between anybody (person-to-person), anything (person-to-machine, machine-to-machine), wherever the devices are and whatever electronic/wireless devices need [24, 27].

Internet Sockets are the endpoints for data communication streams and these are used to recognize all the communication links between nodes. Each web socket is a unique combination of local IP address, proper transport communication port, target IP address, target communication port, and type of transport protocol. In a heterogeneous wireless network, when wireless mobile devices change their Radio Access Technology (RAT) or perform vertical handover then by default IP addresses of respective devices changes. When the devices connect to other RAT, the devices consume more resources and create a delay. The change of RAT means that the change of IP address, the change of any parameter in a socket means that disconnects the current socket and connect to the new socket (new communication link). This is the main problem with the current internet connections. To overcome this critical issue in a $5 \mathrm{G}$ network, IP addresses of the source and destination devices must be fixed and unchanged in case of any interoperability.
By this way, it will ensure the handover transparency and preserve the proper layout data packets.

In order to accommodate the high demand for network data traffic, a new $5 \mathrm{G}$ network will feature by the frequency reuse, an extreme number of wireless devices, and multiple base stations, etc. Therefore, the system requires more attention to enhance interference management techniques. There are different types of interferences that can badly affect the $5 \mathrm{G}$ wireless network [25]. Other-Cell Interference, the devices which are at the edge of the cell usually suffer two types of interference. The first is self-cell interference from the other devices in the same cell and the second is other-cellinterference from the devices of other cells. Cross-tier Interference: in a multi-tier cellular communication network, the interference from one tier to another is indicated as crosstier interference, it degrades the system performance. Intersystem Interference: the interferences from the signal transmitted by the other system. To control these types of interferences there are some interference coordination techniques like beamforming, power control, user scheduling, and advanced receiver techniques. In a $5 \mathrm{G}$ network, carrier aggregation and use of the multi-RAT will be directed to an enhanced mobile spectrum usage. Different radio technologies will empower higher spectrum efficiency by using small cells and massive MIMO. The frequency allocation and mobile infrastructure both can improve the statistical multiplexing and enhance the density of the base station per user. These techniques require a shared backhaul network for the mobile user that further increase the network heterogeneity.

To enhance the current wireless technologies, this paper presents a novel 5G network architecture. Where a whole macro network area is divided into the microcells which are further sub-divided into the smaller cells in order to achieve the full network coverage. The required base stations are equipped with massive MIMO-OFDMA antennas. To prevent the disconnections of vertical handover and delay due to the interoperability, this technique proposed IP-based vertical handover. Where the devices will establish a new RAT link before disconnecting the current link.

\section{A NOVEL ARCHITECTURE FOR 5G Ultra DENSE Heterogeneous Cellular NeTwork}

The proposed 5G network architecture considered the dense heterogeneous wireless network with the deployment of wireless base stations having massive MIMO with OFDMA antennas. The whole Macro Area Network (MAN) into the MicroCells (MCs), each MicroCell has covered by the powerful Micro Base Station (MC_BS). There is one powerful Macro Area Network Base Station (MAN_BS). All the MC_BS are connected with the MAN-BS, which is connected to the core network. The MC is further divided into the Small Cells (SCs) in order to achieve the full network coverage. Each SC has its Base Station (SC_BS), SC is used to upgrade the performance of MAN by offloading the data traffic generated in the network. The degree of integration determines the network performance. The proposed architecture is IP based because it prevents the vertical handover disconnections between mobile devices in case the cells or RAT is changed. The proposed architecture allows switching between different 
RATs. The general layout of the proposed 5G ultra-dense heterogeneous network is shown in Fig 2.

The whole network architecture represents a multi-tier backhaul communication system of the ultra-dense network. The whole ultra-dense area is covered by the small cells as the small cells are designed to fill up the uncovered gaps in microcellular areas. These are physically smaller and covered limited areas. The most important question is that how much densification an SC-BS can cover? The whole micro area has divided into multiple SC where each cell has its own base station. As the microcell is an ultra-dense area, therefore in order to provide full coverage, all the wireless devices are connected to its nearest SC-BS. The base stations are equipped with Massive MIMO antennas therefore, a single base station has an ability to connect maximum users. The $P_{\text {loss }}$ factor is used as a function of the probability of loss of the distance between transmitter and receiver (wireless mobile device- SCBS). The probability loss $P_{\text {loss }}$ is derived as:

$P_{\text {loss }}=\min (\boldsymbol{Q} / d, 1) *\left(1-e^{-\frac{d}{2} * \mathbb{Q}}\right)+e^{-\frac{d}{2} * \mathbb{Q}}$

Where $d$ is the distance between the mobile device and it's serving base station. According to the model, within the specified $0 m$ theta meter devices connects to the base station. Within the specified limit the $P_{\text {loss }}$ is 1 . The devices positioned up to the $Q m$ from the SC_BS has guaranteed a strong loss, therefore, the devices connect to the other SC_BS, which is in the proximity.

In order to establish a backhaul data communication link, SC_BS selects the neighboring MC_BS. An SC_BS has to satisfy the following two conditions:

1) The distance between transmitter and receiver is less than or equal to the radius rd of SC_BS,
2) The distance between SC_BS and MC_BS is less than the distance between the transmitter and MC_BS.

3) When the distance between MC_BS and SC_BS is less than rd then SC_BS establish a link. If conditions are satisfied as a result, the SC-BS efficiently allocates the available spectrum to the wireless devices. In addition, it overcomes the path loss due to the small distance and performs intense frequency reuse.

The ultra-dense Backhaul Network Capacity $B \_N C$ is estimated by the following formula [26]:

$B \_N C=\frac{S T(n) \times T r}{N(n)}$

Where $\mathrm{n}$ indicates the number of SC_BSs, $S T(n)$ is the simultaneous transmissions in $\mathrm{MC}, \mathrm{Tr}$ is the transmission rate, and $N(n)$ is the average hop number of backhaul data traffic in MC.

The MAN_BS, MC_BS, and SC_BS are equipped with massive MIMO-OFDMA antennas. The control system of base stations has to manage a special module known as frequency module. The frequency module of MC_BS keeps the record of all connected SC-BSs and the frequency module of an SC-BS keeps the record of all the RATs of all connected mobile devices. SC-BSs update their MC_BS and all the MC_BSs of a MAN update their serving MAN_BS. The MAN-BS maintains the database of the whole network and updates frequently. As all the base stations are equipped with massive MIMO antennas, therefore, MC_BS can connect maximum SC_BSs similarly the MAN_BS also can connect and control multiple MC_BSs. In order to control the critical situation, the MC_BS also has the ability to provide direct links to mobile devices. Finally, the MAN_BS connects with the core network.

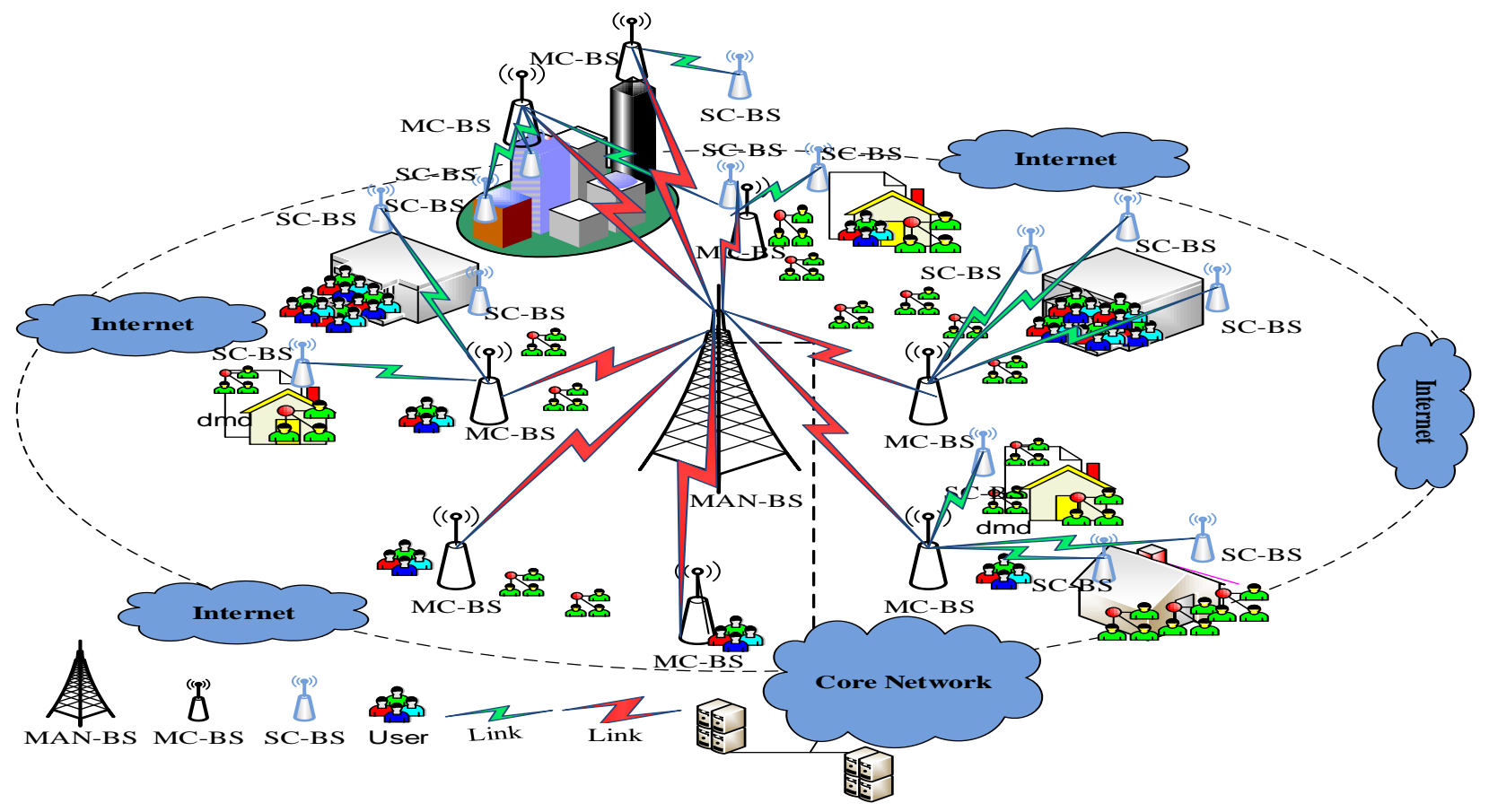

Fig. 2. General Layout of the Proposed 5G ultra-Dense Heterogeneous Network 


\section{A. Massive Multi-Input Multi-Output with Orthogonal Frequency Division Multiple Access (Massive MIMO- OFDMA) Technology}

In the proposed architecture, the BSs are equipped with Massive MIMO with OFDMA antennas as shown in Fig 3. The antennas of MAN_BS, MC_BS and SC_BS are using spatial multiplexing with Orthogonal Frequency Division Multiple Access (OFDMA) modulations. The spatial multiplexing transmits multiple streams of data on the same downlink resource block. These streams have the ability to link one or more wireless devices. All receiver antennas can accept the streams from all the transmit antennas. Spatial Multiplexing Matrix CMM is given in equation 5.

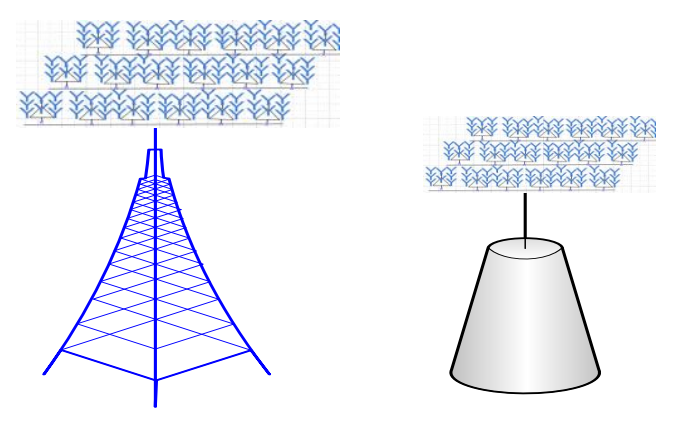

Fig. 3. Base Stations Having Massive MIMO Antennas.

$$
C M M=\begin{array}{cccccc}
C 11 & C 12 & \ldots & C 1 n & \\
C 21 & C 22 & \ldots & C 2 n & \downarrow R n \\
\vdots & \vdots & & \vdots & \\
C n 1 & C n 2 & \ldots & C n n &
\end{array}
$$

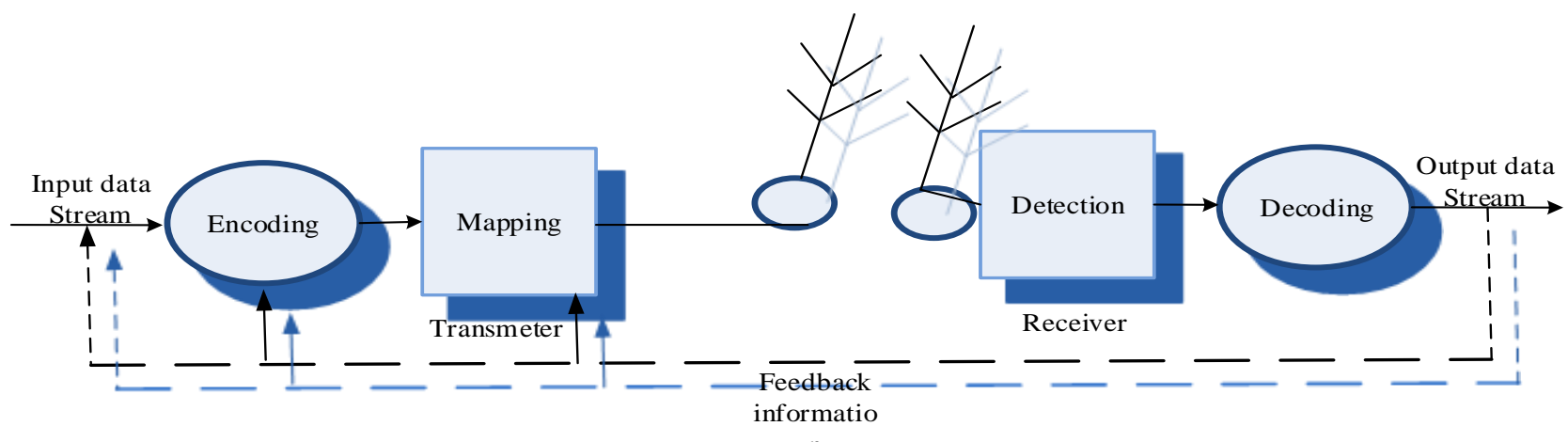

Fig. 4. The General Structure of MIMO Structure.

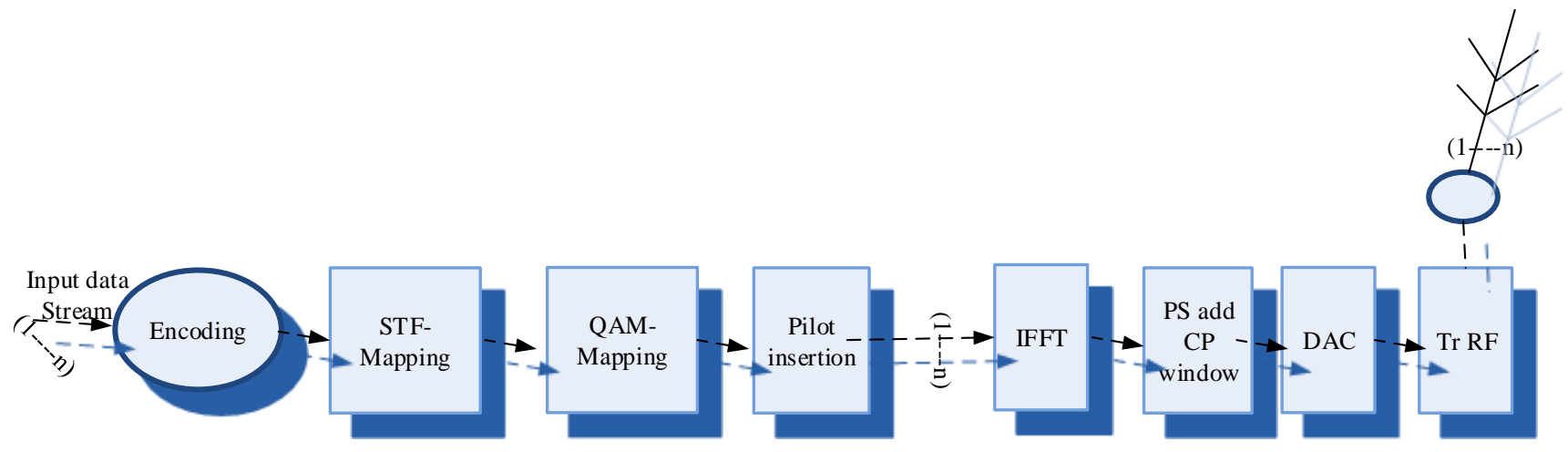

Fig. 5. Massive MIMO-OFDMA Transmitter. 
The massive MIMO-OFDMA receiver is used to represent the digital representation of receiving signals $\mathrm{N}-\mathrm{Re}$. In a next step, the receiver arranges the frequency and gets the symbol timing according to the sequence. All the receiver branches execute the synchronization process together. For an efficient frequency synchronization of multiple branches, it helps to keep all the branches on one side of a link connected to the same local oscillator in a heterodyne scheme. Once the synchronization process completed, it eliminates the guard time known as Cyclic Extension CE (equal to the last part of the OFDM symbol) that eradicates the channel interference. Subsequently, it performs a Fast Fourier Transform (FFT) for each receiver branch. The Fourier analysis changes a signal from its original domain (space or time) in the frequency domain. In order to recover, data streams the STF detection and decoding is executed. In order to track the phase shift, pilot symbols are included in every MIMO-OFDMA data symbol on the predefined subcarrier. Finally, the data streams are combined and STF de-mapping and decoding are executed on transmitted data streams and get the output data stream.

\section{B. IP-based Interoperability in Ultra-Dense Heterogeneous Wireless Network}

When a device connects to the new RAT, first it has to disconnect the current link and establish a new link with the new RAT and IP address. This is time-consuming and inflexible way therefore to overcome this deficiency a new handover technique is proposed. The proposed 5G heterogeneous wireless network has the ability to control multiple RATs at the same time. All the available radio technologies are responsible for handling the wireless device mobility and handover while the user of the terminal device makes the final selection among different RATs. Therefore, all the wireless devices must contain an interface to switch between different RATS. The proposed architecture considers the vertical handover, where wireless devices can perform switching between available RATs.

All the base stations have a built-in control system with some special modules, one of them is Frequency Module FM. This FM is responsible for allocating frequencies to all other connected wireless devices and keeping the updated record. All the wireless devices (connected to the network) must have an interface for using different RATs so the devices can easily select the desired RAT and connect. The FM of SC_BS updates the MC_BS about the frequencies of all connected wireless devices and FM of MC_BS further updates the MAN_BS. If a wireless device wants to switch from one RAT to the other than the control system of a base station manages the handover links. The wireless device chooses the new RAT as displayed in its interface and the wireless device transmits an auto message to the base station where the control system establishes the new link with the desired RAT and then disconnect the previous RAT link. In this case, the interoperability between RATs is done on the network protocol layer that is commonly used by all the RATs. As shown in Fig 6 , an IP based $5 \mathrm{G}$ wireless mobile network. The IP technology is considered to make sure enough control data for the efficient routing of IP packets between wireless devices and servers on the internet.

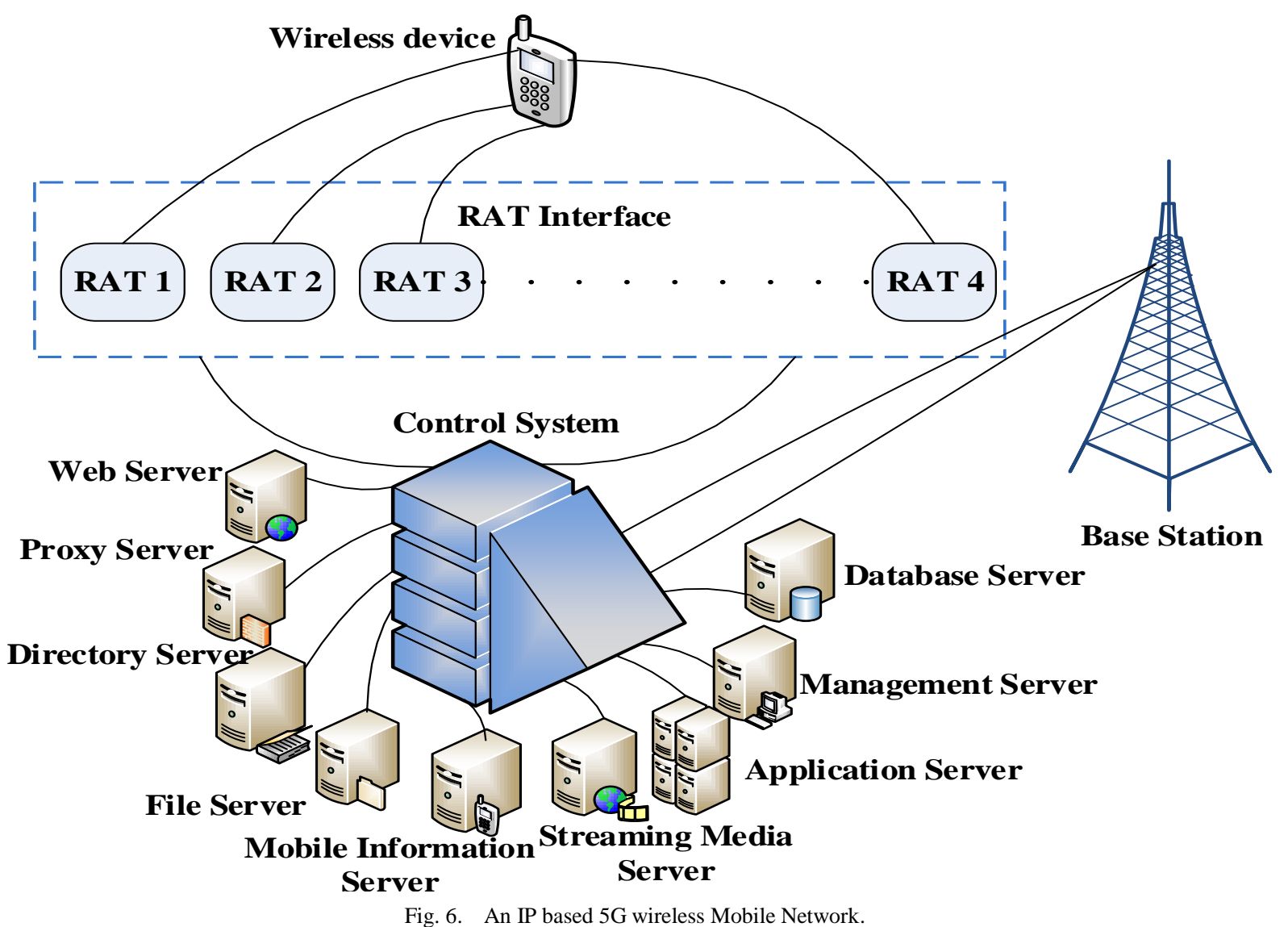

www.ijacsa.thesai.org 


\section{Functionalities of the Wireless Terminal Device}

There are three stages (RAT discovery, RAT selection, and RAT handover) of RAT interoperability. The wireless terminal device must be capable of detecting, selecting and activating the RAT links. The vertical handover process goes through the normal procedure within the specified time in a seamless manner. Like base stations, the wireless devices also have some functional modules, the functional modules are shown in Fig 7. The responsibility of the Database Administration Module (DAM) is to keep the record of connected base stations. A list of neighboring base stations, available RATs, RAT's policies, updated status of RATs, periodically monitored Received Signal Strength (RSS) from the base station, device mobility, and its status. The Network Interface Module (NIM) is responsible for identifying the available RATs on the wireless terminal device and performing switching between different RATs. It monitors the status of each RAT and updates the status during handover. The statuses of all RATs are updated by using a scan, idle, active, turn off and sleep modes. The Mobility Administration Module (MAM) is responsible for handling the mobility and connections of wireless terminal devices. In case a wireless device moves out of the radio range of one small cell to the other or even moves out from one microcell to the other or from one macro area network to the other. The MAM efficiently handle the mobility events and immediately updates the status. Finally, the DAM status is updated.

A fully network connected terminal device is considered. When a terminal device needs to switch from its current RAT, it opens the RAT interface and selects the desired RAT. It updates the DAM to change the mode of the selected RAT. After selecting the Target RAT, the DAM calculates the RSS from its SC-base station and send a link request to the base station. The RSS is calculated to make sure either the terminal device is in the radio range or not. Each RAT has a pre-defined threshold $(t h)$ value for RSS. Whenever a wireless device has to switch its RAT first it calculates the RSS which automatically updates in DAM. If the condition is true, then a terminal device performs the handover. The RSS is calculated by using the following equation.

$R S S_{\text {Target } R A T} \geq R S S_{\text {th,Target } R A T}$

Where RSS Target RAT is the RSS that the terminal device receives from the SC_BS and the $R S S_{t h}$, Target RAT is the RSS threshold value of the Target $R A T$.

The SC_BS sends an acknowledgment (Ack) to the terminal device that shows the connection has been established. After receiving the Ack the terminal device connects with T_RAT and changes the mode. Subsequently, it disconnects the previous RAT and updates its current mode. Thus, the terminal device connects to a new RAT by performing a successful IP based vertical handover, therefore it updates its CS_BS by sending an ACK. Finally, an update is sent to the MC_BS. The wireless terminal device IP based vertical handover procedure is explained in Fig 8. Traditionally, wireless devices linked with a base station may decrease the performance as a mobile device moves away from its base station. In the proposed $5 \mathrm{G}$ network architecture, the DAM of end devices monitors the states and update the database. Therefore, a device having a weak signal strength automatically connects to the neighboring associated base station.
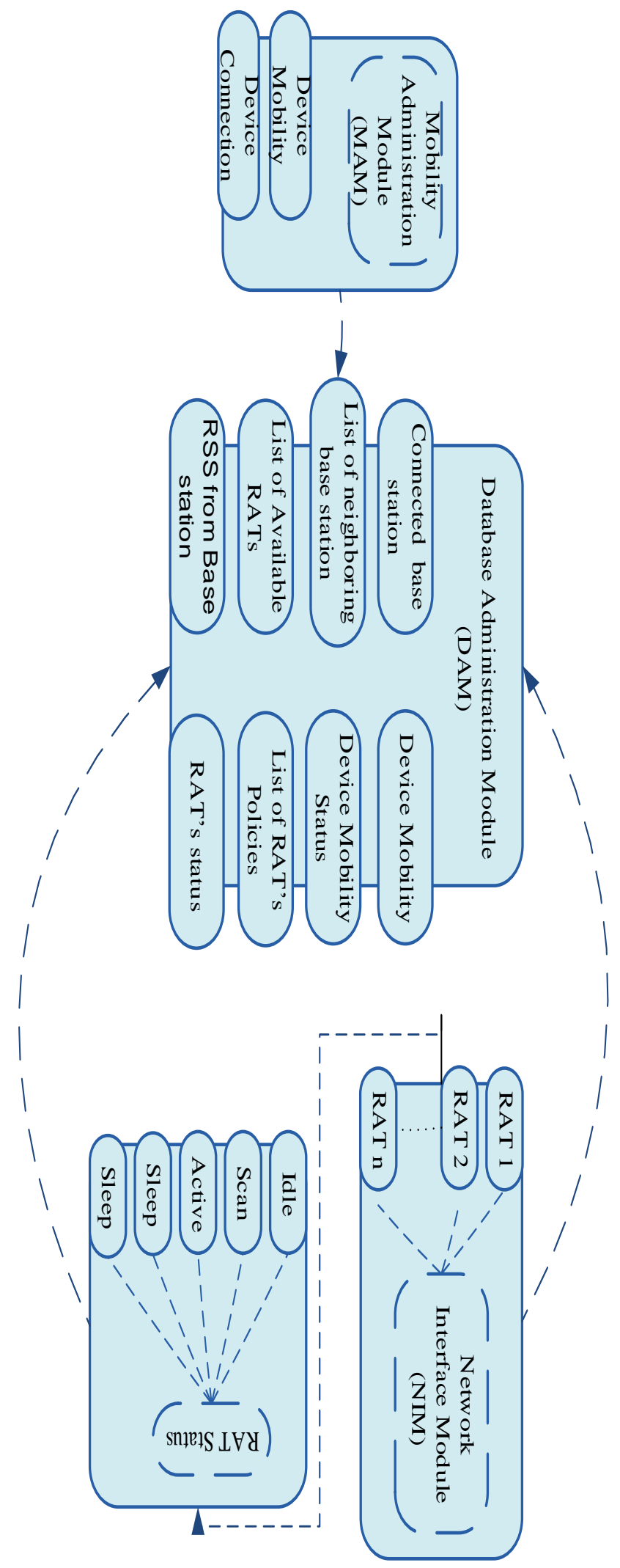

Fig. 7. Functional Modules of wireless Terminal Device. 
(IJACSA) International Journal of Advanced Computer Science and Applications,

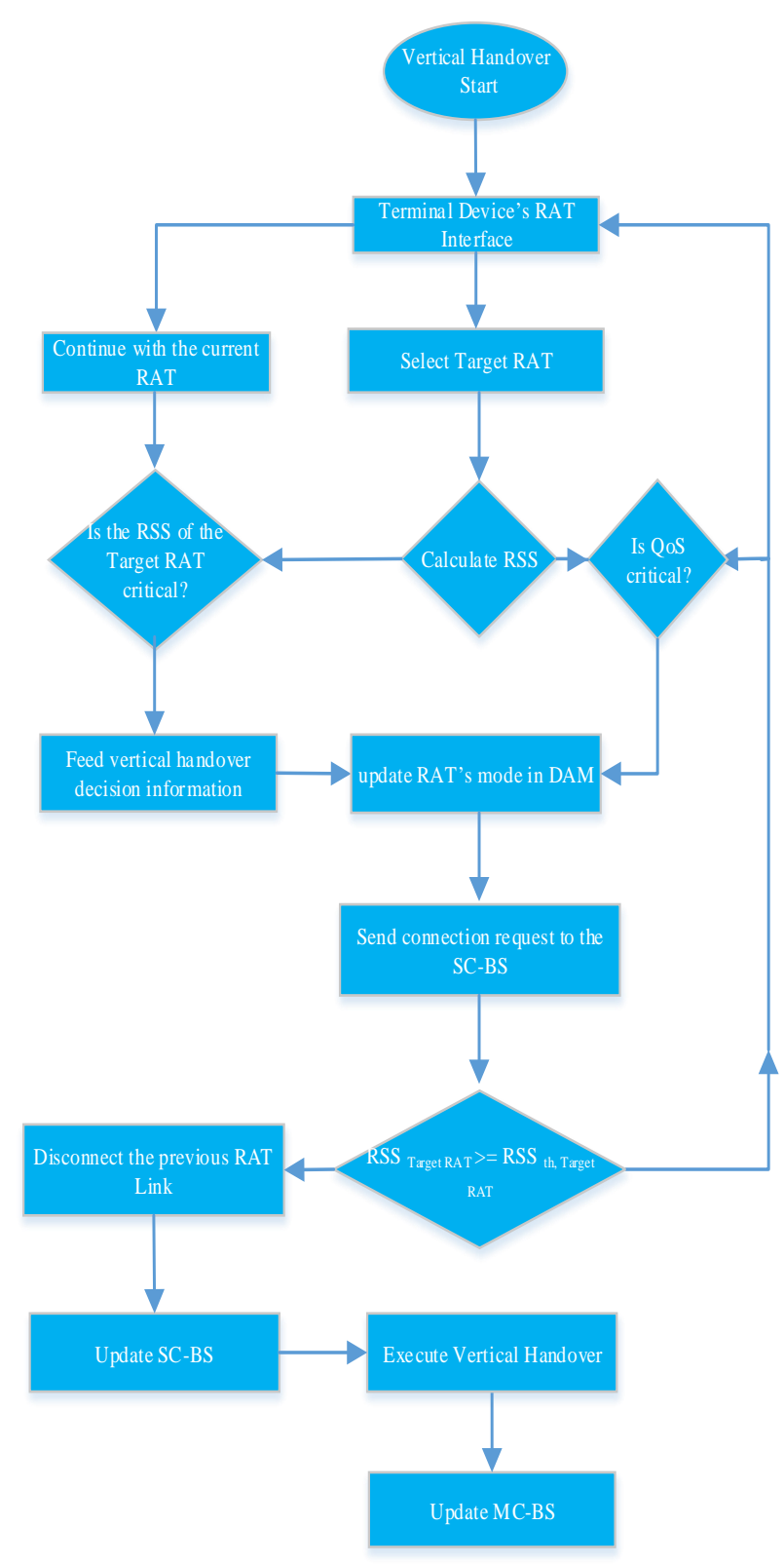

Fig. 8. IP based Vertical Handover of a Terminal Device.

\section{RESULTS AND DISCUSSIONS}

This section evaluates the performance of the proposed architecture and assesses how the network coverage and energy efficiency changed with the macro, micro, and small cells' distribution. The cells' deployment is independent and follows the Uniform and Poisson random measure. The performance of the proposed architecture is evaluated in the OPNET simulator. The simulation area is set to $300 \times 300 \mathrm{~m} 2$ all the base stations are located in the center of the cell whereas the user equipment is randomly located around the base stations. The simulation parameters are listed in table 1 . The radius of base stations SC_BS is set as the $(100 \mathrm{~m}, 200 \mathrm{~m}), \mathrm{MC} \_\mathrm{BS}$ radius $(300 \mathrm{~m}$, $500 \mathrm{~m}$ ) and MAN_BS is $1 \mathrm{~km}$.
TABLE I. SIMULATION PARAMETERS

\begin{tabular}{|l|l|}
\hline Parameters & Values \\
\hline Area & $300 \times 300 \mathrm{~m} 2$ \\
\hline SC_BS radius & $100 \mathrm{~m}, 200 \mathrm{~m}$ \\
\hline MC_BS radius & $300 \mathrm{~m}, 500 \mathrm{~m}$ \\
\hline MAN_BS & $1 \mathrm{~km}$ \\
\hline Traffic mode & CBR \\
\hline Per channel bandwidth & $100 \mathrm{MHz}$ \\
\hline Bandwidth 2 & $20 \mathrm{MHz}$ \\
\hline Scheduling Algorithm & Proportional fair \\
\hline Number of MAN-BS & 3 \\
\hline Number of MC-BS & 9 \\
\hline Number of SC-BS & 55 \\
\hline
\end{tabular}

The network energy efficiency is a key constraint that limits the $5 \mathrm{G}$ network's densification. The energy consumed by the SC-BS is consist of Embodied Energy (EE) and Operational Energy (OE). The EE is the power utilized by all the process related to the $\mathrm{BS}$ implementation. Whereas the $\mathrm{OE}$ is the energy utilized by the operations of backhaul within the network lifetime Time To Live (TTL), which is expressed as:

$O E=B S O P * T T L$

Where BSOP is the BS operational power and it is considered as a linear function of SC_BS. It is defined as $B S O P=A * P_{T r}+\mathrm{B}$, where $P_{T r}$ is transmission Power, $A=$ $7.90 \mathrm{~W}$ and $B=72 \mathrm{~W}$. In order to clarify the derivation model, $P_{T r}$ of a BS is normalized as $P_{N r}=1.5 \mathrm{~W}$ and BS backhaul throughput $\theta$ is considered as $1 \mathrm{~Gb} / \mathrm{s}$. The average throughput $A_{T h}$ is calculated by:

$P_{T r}=P_{N r} \times\left(A_{T r} / \theta\right)$

The operating power of BS has calculated as below:

$B S_{o p}=A \times P_{N r} \times\left(A_{T r} / \theta\right)+B$

The Backhaul Energy Efficiency B_EE is obtained through:

$B_{E E}=\frac{B_{-} N C}{n \times\left(B S_{-} E C\right)}$

Where $B \_N C$ is Backhaul Network Capacity and $B S \_E C$ is Backhaul Energy Consumption.

The energy efficiency is analyzed in Fig 9 and 10 with Uniform and Poisson distribution. At the start, the energy efficiency was improving with the increasing number of small cells then it starts decreases after it reaches the threshold value. With the static number of MAN_BSs, the energy efficiency improves with the increase of radius. With the fixed radius, backhaul energy efficiency with respect to the average of SC_BSs' throughput is explained in figures. 


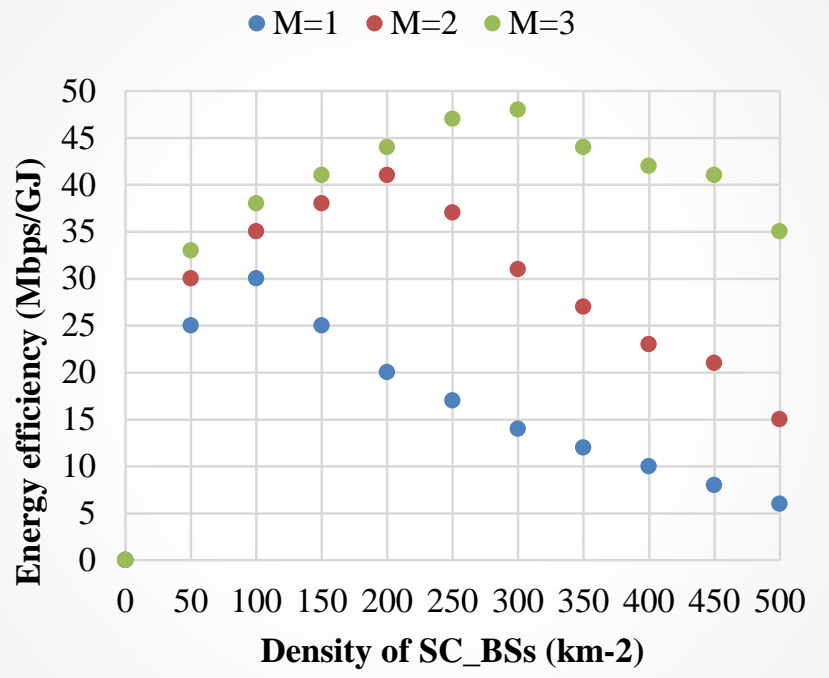

Fig. 9. Energy Efficiency Vs. Density of SC_BSs with Uniform Distribution.

- $\mathrm{M}=1 \quad \mathrm{M}=2 \cdot \mathrm{M}=3$

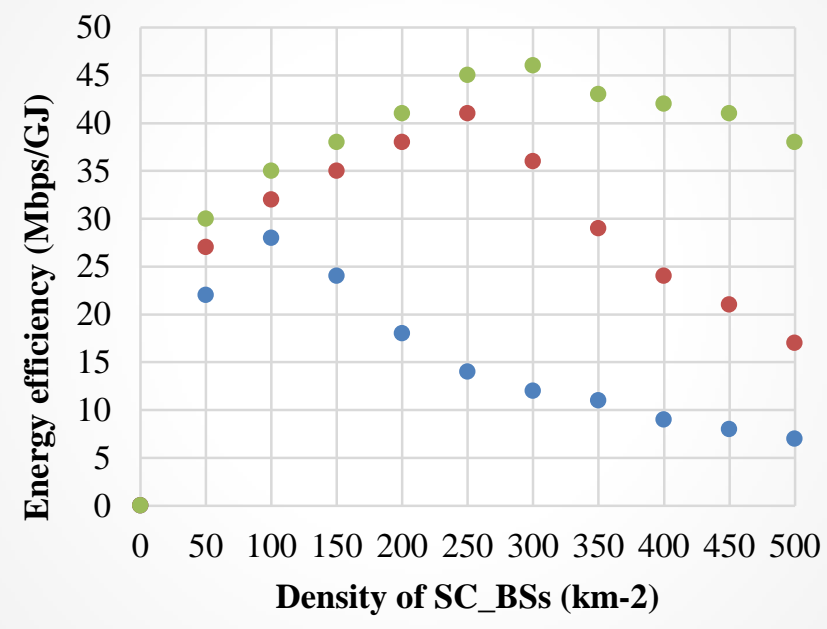

Fig. 10. Energy Efficiency Vs. Density of SC_BSs with a Poisson Distribution.

As a microcell is an ultra-dense area, therefore, to get the full network coverage proposed technique to divide it into the SCs. All the active MTs in a single cell connect to the respective $\mathrm{BS}$ where $\mathrm{BSs}$ are equipped with the MIMO antennas. With the fixed SC radius, the B_NC with respect to the number of SCs is explained in Fig 11, it was observed that the B_NC increases with the increase of a number of small cells. When B_NC touched its maximum limit, it gradually decreases with the increase of SC. In Fig. 12, the average number of simultaneous transmission is found as 19,25 , and 29 when the small cell radius is set as $300 \mathrm{~m}, 200 \mathrm{~m}$, and 100 $\mathrm{m}$ correspondingly. The backhaul network capacity increases with the increase of simultaneous transmissions. When the average number of simultaneous transmission and the number of SC is fixed the backhaul network capacity start decreasing.
From the simulation results, it has observed that when the average simultaneous transmissions are larger than the predefined threshold value then backhaul capacity get the constant value. This simulation output helps to design the ultradense cellular network.

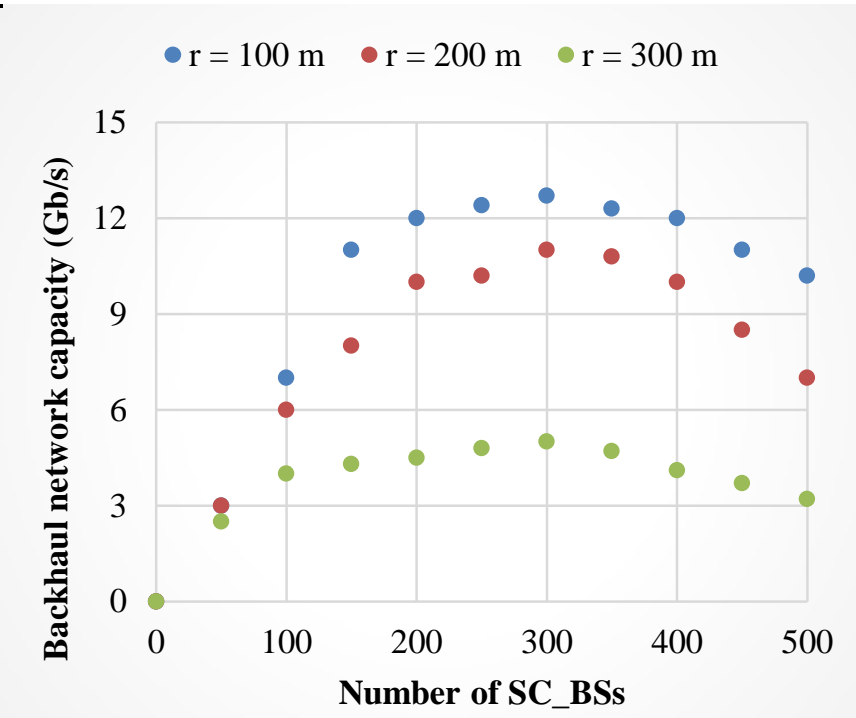

Fig. 11. Number of SC_BSs vs. Backhaul Network Capacity.

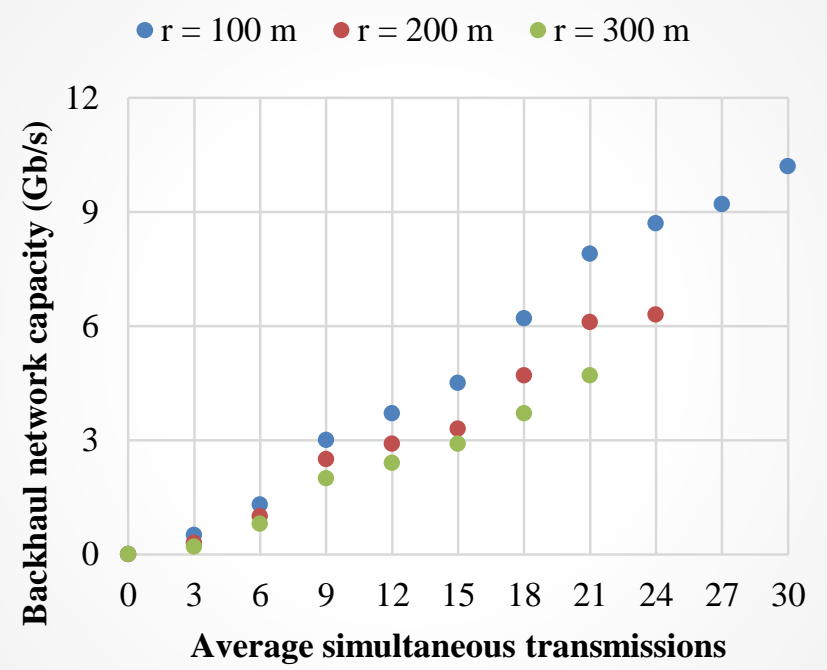

Fig. 12. Average Simultaneous Transmissions Vs. Backhaul Network Capacity.

In the proposed architecture, every BS is equipped with an FM, which has to allocate the frequencies to the other connected MTs. An MT can easily select the desired RAT from its interface. An FM of SC_BS periodically informs the respective MC_BS about the frequencies of all the connected MTs and it further updates the MAN_BS. If a MT needs to switch from its C_RAT first it selects a T_RAT from the available RATs as displayed in the interface. The MT sends an auto message to the BS where the control system manages a new link with the T_RAT and disconnect the C_RAT links. In Fig. 13, the backhaul network capacity with respect to the 
number of SC_BSs is shown, MC-BS starts increasing with the increase of $\mathrm{SC}_{-} \mathrm{BSs}$ and with the increase of density of a small cell. When the density of an SC is equal to or greater than the defined threshold value then the capacity reaches a static value. The backhaul network capacity is analyzed with the Uniform and Passion distribution. When the backhaul network capacity with the Uniform distribution of $\mathrm{SC}_{-}$BSs attains the static values as in Fig 14, the density thresholds of a small number of MAN_BSs $(\mathrm{M})=3,2$ and 1 , respectively. When the backhaul network capacity with the Poisson distribution of small cell BSs achieves the static values in Fig.14, the density thresholds of small cell BSs are 0-500 which relates to the number of MAN_BSs $(\mathrm{M})=3,2$ and 1 .

$\bullet \mathrm{M}-1 \bullet \mathrm{M}-2 \cdot \mathrm{M}-3$

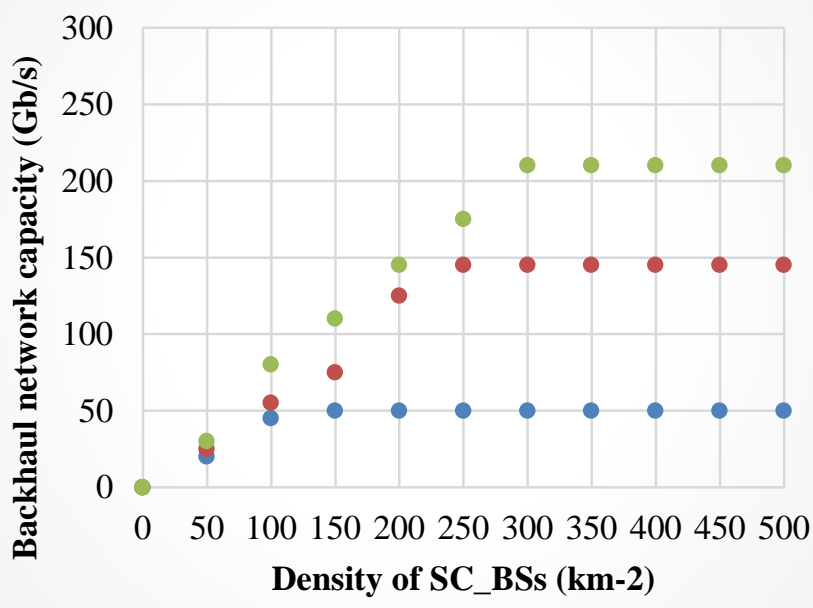

Fig. 13. Backhaul Network Capacity vs. Density of SC_BSs with Uniform Distribution.

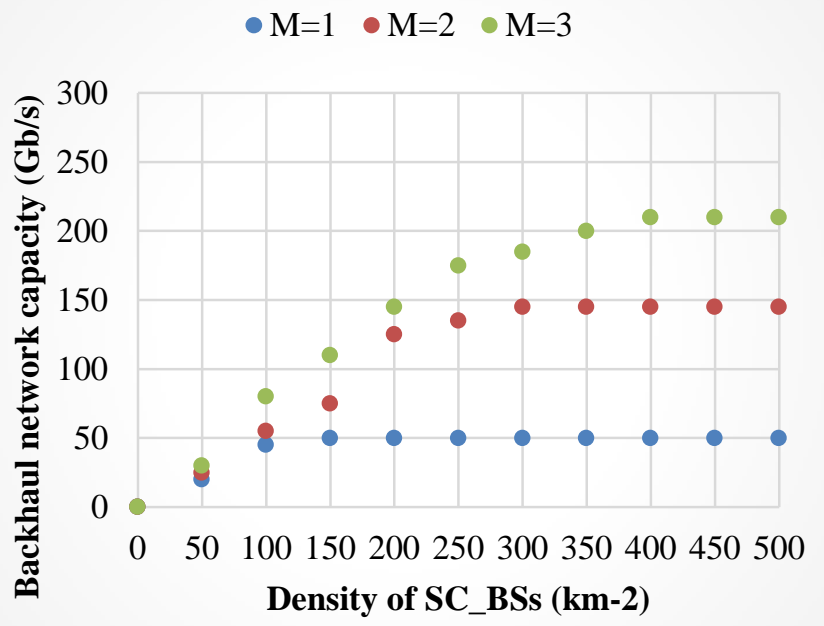

Fig. 14. Backhaul Network Capacity vs. Density of SC_BSs with a Poisson Distribution.

\section{Future ChaLlenges}

The increasing use of the ultra-dense cellular network is motivated by the massive MIMO-OFDMA and IP based vertical handover, the distributed network architecture is a reasonable solution. In spite of $5 \mathrm{G}$ network benefits, the small cell densification opens up new research directions. There are some potential challenges of scheduling, routing relay optimization, interference, and allocation of best massive MIMO antennas etc. Scheduling is considered an efficient technique to utilize the available frequency. On the other side, the proportional fair scheduler can also be used to improve the throughput with a large number of wireless mobile devices. A $5 \mathrm{G}$ distributed wireless network needs to carefully select a routing path between the source and destination devices. If base stations are equipped with the complex massive MIMO antennas, then there is highly required reasonable allocation of massive MIMO antennas for achieving efficient transmission.

A $5 \mathrm{G}$ wireless network will handle the huge amount of data traffic of the ultra-dense wireless network but interference can be a critical challenge. Therefore, the system needs more attention to implementing the interference avoidance techniques. The use of high frequency, the highest data rate, minimum delay, quick response time, complex antennas, implementation of multiple RATs in a single wireless device pays attention to the development of advanced base stations and wireless devices. The implementation of the $5 \mathrm{G}$ network is not limited to the hardware, but it highly requires the implantation of new protocols and algorithms.

\section{CONCLUSION}

Ultra-dense wireless networks are still considered as a complement to the cellular wireless network with an efficient architecture. The massive MIMO antennas facilitate the 5G ultra-dense cellular networks to be scattered in all wireless cellular set of connections. In this paper, a novel $5 \mathrm{G}$ wireless network architecture is presented with two main aspects of massive MIMO-OFDMA and IP based vertical handover. The proposed architecture divides the whole macro area network into the microcells and each micro-cell consists of multiple small cells. All the cells are manipulated by their adjacent massive MIMO-OFDMA base stations. The heterogeneity and small-sized cell base stations provide full network coverage. The proposed architecture performs switching between different RATs. All the devices are designed with a multiple RAT interface from where the user can select the desired RAT and perform vertical handover. In order to illuminate the handover delay and improve the service continuity, the vertical handover is IP based. The advantage of using IP based vertical handover is establishing new connections with the same IP address before disconnecting the previous links.

\section{REFERENCES}

[1] A. Zakrzewska, S. Ruepp, and M. Berger., "Towards converged 5G mobile networks - Challenges and current trends," in Proc. ITU Kaleidoscope Academic Conference. 2014. p. 39-45,

[2] S. Talwar, et al., "Enabling technologies and architectures for 5G wireless," in IEEE MTT-S International Microwave Symposium (IMS),. 2014. p. 1-4,

[3] ZoraidaFrias and JorgePérez Martínez, 5G networks: Will technology and policy collide?. Telecommunications Policy, Elsevier, 2017.

[4] J. Bernardos, et al., An architecture for software-defined wireless networking. Wireless Commun. IEEE., 2014. 3(21): p. 52-61.

[5] E. Piri et all, 5GTN: A test network for 5G application development and testing, European Conference on Networks and Communications (EuCNC), Athens, p. 313-318.

[6] J. P Romero, et al., "Radio Resource Management Strategies in UMTS", 2005, John Wiley \& Sons, New York, USA. 
[7] A. Ejaz et al., "Recent Advances and Challenges in Mobile Big Data", IEEE Communications Magazine 2018. 56(2): p. 102-108.

[8] Vinicius C. M. Borges, et al., "Aspirations, challenges, and open issues for software-based $5 \mathrm{G}$ networks in extremely dense and heterogeneous scenarios", EURASIP Journal onWireless Communications and Networking, 2015. 164.

[9] S. T. Bakhsh, et al., Cross-layer-based Adaptive Traffic Control Protocol for Bluetooth Wireless Networks, International Journal of Advanced Computer Science \& Applications, p. 102-108, vol 8, June 2017.

[10] S Hong, et al., Applications of Self-interference Cancellation in 5G and Beyond, in Commun. Mag. IEEE 2014. p. 114-121.

[11] WH Chin, Z Fan, and R Haines, "Emerging technologies and research challenges for 5G wireless networks", Wireless Commun. IEEE, 2014. 2(21): p. 106-112.

[12] WH Chin, Z Fan, and R Haines, Emerging technologies and research challenges for 5G wireless networks. Wireless Commun. IEEE., 2014. 2(12): p. 106-112.

[13] C-X Wang, et al., Cellular architecture and key technologies for 5G wireless communication Networks, in Commun. Mag. IEEE.. 2014. p. 122-130.

[14] E Björnson, et al., Multiobjective signal processing optimization: the way to balance conflicting metrics in 5G systems, in IEEE Signal Processing Magazine. 2014. p. 14-23.

[15] Noha Hassan and Xavier Fernando, Massive MIMOWireless Networks: An Overview, Electronics, 2017: p. 29.

[16] Stefano Ruffini, et al., A Novel SDN-Based Architecture to Provide Synchronization as a Service in 5G Scenarios, in IEEE Communications Magazine, 2017.
[17] T Bogale, et al., "User scheduling for massive MIMO OFDMA systems with hybrid analog-digital beamforming", in IEEE ICC. 2015.

[18] W Liu, A Zappone, and C Yang, "Global EE optimization of massive MIMO systems", in IEEE SPAWC. 2015.

[19] Qian Wang, et al., 5G MIMO Conformal Microstrip Antenna Design, Wireless Communications and Mobile Computing, 2017. 2017: p. 11.

[20] M., N.H., et al., Massive MIMO for Fifth Generation (5G): Opportunities and Challenges, in International Conference on Computer and Communication Engineering (ICCCE),. 2016: Kuala Lumpur,

[21] G. Xiaohu, et al., "5G Ultra-Dense Cellular Networks". IEEE Wireless Communications, 2015.

[22] M. Fadi et al., "Energy Efficiency Perspectives of Femtocells in Internet of Things: Recent Advances and Challenges", IEEE Access, 2017, p. 26808-26818.

[23] Osseiran. A., et al., "Scenarios for 5G mobile and wireless communications: the vision of the METIS project," in Communications Magazine, IEEE,. 2014. p. 26-35.

[24] C-X Wang, et al., Cellular architecture and key technologies for 5G. wireless communication Networks. Commun. Mag,, 2014. 52(2): p. 122130.

[25] Lu. Yang and Wei. Zhang, Interference Coordination for 5G Cellular Networks. SpringerBriefs in Electrical and Computer Engineering, Springer, 2015.

[26] G. Mao, et al., "Towards a Simple Relationship to Estimate the Capacity of Static and Mobile Wireless Networks", IEEE Trans. Wireless Commun. Aug. 2013. 12(8): p. 3883-3895.

[27] I. Muhammad, et al., reconfigurable scatternet formation and maintenance scheme with heterogeneous services for smart Bluetooth devices, Sustainable Cities and Society, vol 36, p. 589-599, 2018. 